\title{
CAN MUSCLE FATIGUE IN WOMEN BE INFLUENCED BY KNEE EXTENSION TASKS IN DIFFERENT RANGES OF MOTION?
}

original paper

() Wroclaw University of Health and Sport Sciences

DOI: https://doi.org/10.5114/hm.2022.107981

\section{GUSTAVO FERREIRA PEDROSA ${ }^{1,2}$, FERNANDO VÍTOR LIMA ${ }^{1}$, RODRIGO CÉSAR RIBEIRO DINIZ ${ }^{1}$, MARINA GURGEL SIMÕES ${ }^{1}$, MARIANO REZENDE PEREIRA ${ }^{1}$, STAYCE MOREIRA FORTUNATO ${ }^{1}$, GABRIEL ROCHA DE OLIVEIRA AMORIM ${ }^{1}$, MAURO HELENO CHAGAS ${ }^{1}$}

${ }^{1}$ Weight Training Laboratory, School of Physical Education, Physiotherapy and Occupational Therapy, Federal University of Minas Gerais, Belo Horizonte, Brazil

${ }^{2}$ Brazilian Air Force, Aeronautical Instruction and Adaptation Centre, Lagoa Santa, Brazil

\begin{abstract}
Purpose. The present study aimed to compare the strength performance in the one-repetition maximum (1RM) test with a knee extension machine among different ranges of motion (ROMs), and to compare the force reduction after the performance of a dynamic exercise configured with different ROMs.

Methods. Nine women (mean \pm standard deviation: age: $24.2 \pm 3.5$ years; height: $166.5 \pm 4.1 \mathrm{~cm}$; body mass: $68.35 \pm$ $4.14 \mathrm{~kg}$ ) with no strength training experience and no history of injury performed (cross-over design) tests of 1RM with a knee extension machine in the following ROMs: $100-65^{\circ}$ of knee flexion (INITIAL $L_{\text {ROM }}$ ), $65-30^{\circ}$ (FINAL ROM ), and $100-30^{\circ}$ $\left(\mathrm{FULL}_{\mathrm{ROM}}\right)\left(0^{\circ}=\right.$ knee full extended). Further, the volunteers performed, in each ROM, 3 sets of 7 repetitions at $60 \%$ of $1 \mathrm{RM}$ (specific to ROM assessed) with 3-minute rests between sets with 2 seconds for concentric and eccentric phases. Before and 2 minutes after the training, the maximum torque values at $100^{\circ}$ and $30^{\circ}$ of knee flexion were registered to calculate the force reduction.

Results. The ANOVA test identified that the maximum torque pre-training values were greater than the post-training values $(p=0.02)$, and a greater torque reduction occurred at $30^{\circ}$ of knee flexion than at $100^{\circ}(p=0.001)$.

Conclusions. The results suggest that ROM may influence maximum strength performance, and the force may reduce similarly along the angles.
\end{abstract}

Key words: muscle strength, range of motion, joint, fatigue, torque

\section{Introduction}

Range of motion (ROM) can be defined as the angular distance travelled by a body joint [1]. Across a ROM, the muscle varies its length [2], being able to influence instantaneously its torque production capacity [3]. In addition, the resistance torque may also be altered along the ROM by increasing or diminishing the external arm moment [4]. Considering that the torque production capacity and the resistance torque are influenced by ROM, few studies compared the maximum strength performance with the one-repetition maxi- mum (1RM) test using different ROMs for the same exercise [5-7].

In these studies, the full ROM (FULL ${ }_{\mathrm{ROM}}$ ) and the final partial ROM (final half of the angles of a FULL ROM $_{\text {, }}$ considering the concentric muscle action as reference: FINAL $_{\text {ROM }}$ ) were used for the comparison. The result of this comparison indicated that a greater amount of weight could be lifted with the FINAL ROM $_{\text {in contrast }}$ to FULL $_{\mathrm{ROM}}$. It is worth noting that the exercises used in those studies (squat or bench press) offer greater external arm moment (in the knee and hip for squat, and shoulder for bench press) nearby the beginning

Correspondence address: Gustavo Ferreira Pedrosa, Universidade Federal de Minas Gerais, Av. Presidente Antônio Carlos, nº 6627, CEP: 31270-901, Belo Horizonte, Brazil, e-mail: gustavofpedrosa@gmail.com

Received: October 29, 2020

Accepted for publication: April 27, 2021

Citation: Pedrosa GF, Lima FV, Diniz RCR, Simões MG, Pereira MR, Fortunato SM, Amorim GRO, Chagas MH. Can muscle fatigue in women be influenced by knee extension tasks in different ranges of motion? Hum Mov. 2022;23(3):56-64; doi: https://doi.org/10.5114/hm.2022.107981. 
of the concentric muscle action and not at the end. This may have negatively influenced the maximum weight lifted during the $1 \mathrm{RM}$ test for the FULL $\mathrm{ROM}_{\mathrm{R}}$ in comparison to FINAL $_{\text {ROM }}$ [5]. If the external arm moment is a determining factor for strength performance, then in an exercise with a greater external arm moment at the end of the concentric action, such as with a knee extension machine, the comparison of the 1RM test performance between FULL $_{\text {ROM }}$ and FINAL ROM $_{\text {could }}$ show a similar result, but this reasoning has yet to be confirmed.

Additionally, prior studies [5-7] did not compare the $1 \mathrm{RM}$ test performance with FULL $\mathrm{ROM}_{\mathrm{R}}$ and the initial partial ROM (initial half of the angles of a FULL ROM $_{\text {, }}$ considering the concentric muscle action as reference: INITIAL $_{\text {ROM }}$ ). In the knee extension exercise, perhaps the $1 \mathrm{RM}$ test performance with INITIAL $\mathrm{ROM}_{\mathrm{RO}}$ would be greater than with FULL $\mathrm{ROM}_{\text {, }}$ as the greater external arm moment is located at the final and not at the initial

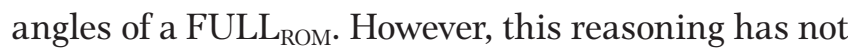
yet been tested. Knowing whether ROM influences the strength performance may help coaches to prescribe specific training loads in order to develop the ROM in which the sport modality occurs. Also, coaches may start the exercise in a determined ROM and finish it in another ROM, as the exercise could keep being performed in the ROM in which the fatigue is lesser, expanding the training volume. In addition, males and females may present different patterns of strength response across the same joint angles [8]. Previous research compared the maximum dynamic strength performance in the FINAL $\mathrm{ROM}_{\mathrm{R}}$ and FULL $\mathrm{FOM}_{\mathrm{RO}}$ with men [5-7]; thus, new studies with women are necessary.

In addition to the external arm moment, muscle length is another variable influenced by ROM. Exercising in different muscle lengths may alter biomechanical and physiological responses [8-11]. Kooistra et al. [9] showed that greater maximum oxygen consumption occurred at a bigger muscle length $\left(90^{\circ}\right.$ of knee flexion $-0^{\circ}=$ full knee extension) than at a smaller muscle length ( $30^{\circ}$ of knee flexion) during isometric contractions. In another study, Kooistra et al. [12] demonstrated that greater time under tension could be held at a smaller than at a bigger muscle length $\left(30^{\circ}\right.$ and $90^{\circ}$ of knee flexion, respectively) in isometric contractions. These results indicate that contractions with a bigger muscle length could be more fatiguing than those with a smaller muscle length, at least in isometric contractions.

However, previous studies [9, 12] did not verify if the muscle fatigue would be also greater at a bigger than at a smaller muscle length after a dynamic exercise performed in different ROMs, such as the INITIAL ROM $_{\text {, }}$

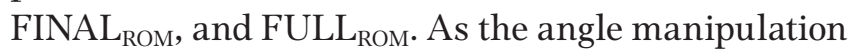
during the muscle contractions can bring different physiological responses, e.g. maximum oxygen consumption levels [9, 12], it is expected that different levels of muscle fatigue may also be found when ROM is manipulated in a dynamic exercise. It has been accepted that muscle fatigue can be identified by a reduction in the ability to produce force $[8,13,14]$. Thus, dynamic contractions in different muscle lengths (i.e., different ROMs) could induce different levels of force reduction. This reasoning, though, has yet to be tested, in particular with women, as this public appears to be more susceptible to muscle injury when submitted into fatigue conditions in comparison with men [8]. Then, the information whether ROM impacts force reduction could help coaches, for example, to prescribe longer or shorter pauses for recovery during the training in an attempt to adjust the objectives desired, considering the muscle fatigue resulting from ROM manipulation.

In addition, some studies have pointed the existence of a specific increase in force production mainly in the angles trained or near them $[5,15,16]$, but the mechanics behind this specific effect has not yet been elucidated. A factor that could be linked to this adaptation would be specific fatigue caused only in the angles trained. This expectation is in accordance with observations by Rhea et al. [6] that strength improvement was specific to joint angles that were sufficiently overloaded. As the overload from training may lead to acute muscle fatigue [14] and chronic adaptations such as strength increase [17], perhaps angle-specific muscle fatigue can be found when the muscle is submitted to overload in a particular ROM. As the force reduction is considered a sign of muscle fatigue $[8,13$, 14], the comparison of force reduction between angles after the performance of a dynamic exercise, as with a knee extension machine, with different ROMs, such as the INITIAL ROM $_{\text {, FINAL }}$ ROM, and FULL ROM $_{\text {, may }}$ provide evidence of an angle-specific fatigue effect that could contribute to explaining the strength increase within the angles trained [17].

Therefore, the present study aimed to compare strength performance in women by a $1 \mathrm{RM}$ test with a knee extension machine among different ROMs, and to compare the force reduction after the performance of a dynamic exercise configured with different ROMs. 


\section{HUMAN MOVEMENT}

G.F. Pedrosa et al., Knee extension tasks

\section{Material and methods}

\section{Participants}

The study sample was composed of 9 women with no strength training experience, or who had not been training for at least a year, and no history of injury (mean \pm standard deviation: age: $24.2 \pm 3.5$ years; height: $166.5 \pm 4.1 \mathrm{~cm}$; body mass: $68.35 \pm 4.14 \mathrm{~kg}$ ). The sample size was calculated with the $G^{*}$ Power (3.1.9.2) software; the recommendations by Beck [18] were followed, as well as a study with a similar purpose [19] that presented an effect size of 0.59 for the absolute torque reduction after an induction, with an a priori statistical power $(1-\beta)$ of 0.8 , a $5 \%$ significance level, 3 groups

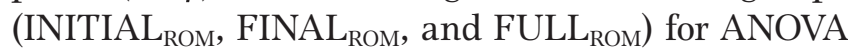
(repeated measures, within factors). Before the participation in the study, the women were informed on the procedures, risks, and benefits of the investigation.

\section{Design and procedures}

This study used a crossover design to compare the $1 \mathrm{RM}$ test performance among 3 different ROM configurations (INITIAL ROM , FINAL ROM $_{\text {, and FULL }}$ ROM) with a knee extension machine, and to compare the force reduction in 2 angles, by torque values, after the execution of 3 training protocols differentiated by ROM (INITIAL $_{\text {ROM }}$, FINAL ROM $_{\text {, and FULL }}$ ROM $)$. Each volunteer presented to the laboratory on 4 different days (experimental sessions 1-4), separated by at least 48 hours (Figure 1).

In the first session, after the assessment of height and body mass with a stadiometer (precision of $1 \mathrm{~mm}$ and $100 \mathrm{~g}$, respectively) (Welmy ${ }^{\circledR}$, São Paulo, Brazil), the participants were positioned on a knee extension machine (Master ${ }^{\circledast}$, Belo Horizonte, Brazil). The angle of the trunk and hip was set at $110^{\circ}$, and the participant's ankle touched the machine cushion at $2 \mathrm{~cm}$ above the ankle medial malleolus. All positions were registered for the following sessions. Afterwards, the volunteers were familiarized with the $1 \mathrm{RM}$ test in 3 ROMs (order balanced among the ROMs). The first ROM was the INITIAL $L_{\text {ROM }}$, and the women had to extend the knee from $100^{\circ}$ to $65^{\circ}\left(0^{\circ}=\right.$ knee full extended). The second ROM was the FINAL ${ }_{\text {ROM }}$, and the women extended the knee from $65^{\circ}$ to $30^{\circ}$. The third ROM was the FULL $\mathrm{ROM}_{\mathrm{RO}}$, and the volunteers extended the knee from $100^{\circ}$ to $30^{\circ}$. Figure 2 shows the 3 ROMs used.

The 1RM test was performed during the first 2 sessions with the aim to familiarize the participants

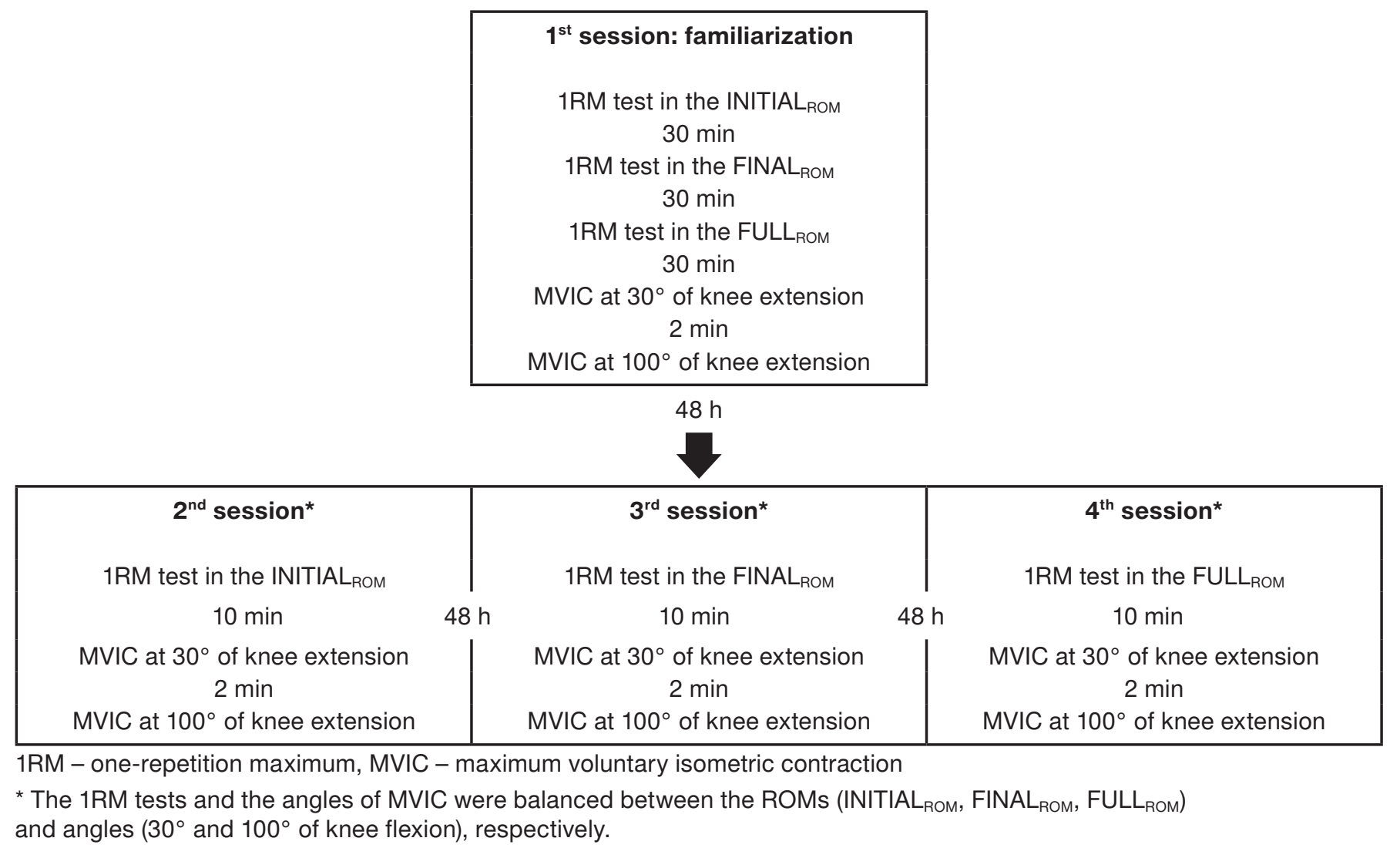

Figure 1. Experimental design 

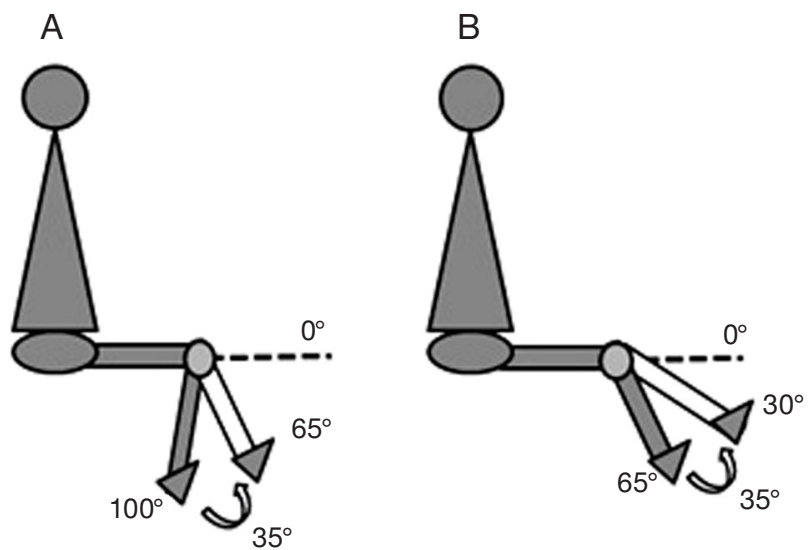

with its procedures and to define the weight (intensity) for the following procedures, respectively. In the first session, the volunteers performed the 1RM familiarization tests in each ROM presented in Figure 1. A 30-minute interval was given between the $1 \mathrm{RM}$ familiarization tests. For all tests, a maximum number of 6 attempts were allowed, in which the weight was gradually increased (minimum of $1 \mathrm{~kg}$ ), with a 3-minute rest between each attempt. When the participant could not perform the concentric action within the determined ROM, the prior weight lifted was recorded, representing the 1RM test result. When the volunteers performed the FINAL $\mathrm{R}_{\mathrm{ROM}}$, the machine weight support was lifted by a manual lifter (jack; Figure 2A) until the desired height that allowed the woman start the concentric action in $65^{\circ}$ of knee flexion without any previous leg acceleration. Ten minutes after the last 1RM familiarization test, the participant was instructed to perform 2 maximum voluntary isometric contractions (MVICs) at $30^{\circ}$ and $100^{\circ}$ of knee flexion with a 2-minute interval between the attempts, aiming at MVIC familiarization. For the MVIC at $30^{\circ}$, the machine weight support was also lifted by the jack until the adequate height.

Additionally, in front of the knee extension machine, a metal apparatus was placed. When the volunteer extended the knee until the desired angle, the metal apparatus was hit by a metallic protractor that was connected to the machine nearby the cushion (Figure 2B). The metal gave the participant a visual feedback denoting the exact moment to end the concentric action during the test, and other procedures were further explained. It is worth noting that for INITIAL $\mathrm{L}_{\mathrm{ROM}}$, a small bell (Figure 2B) was connected to the metal apparatus, as the participant could not see properly when the apparatus was hit by the metallic protractor at this angle. Hence, the women could listen to the bell ring and stop the knee extension to beyond $65^{\circ}$ of knee flexion. In addition, a mirror was placed at the left side
Figure 2. Protocols with different ranges of motion:

(A) INITIAL $\mathrm{L}_{\mathrm{ROM}}$ - initial partial range of motion

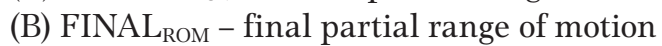

(C) FULL $_{\text {ROM }}$ - full range of motion

of the apparatus to ensure full visualization of all ROMs tested. On the ground and the machine cables, tape marks were glued. The alignment of these tape marks (ground with cable) indicated the end of the eccentric action and the beginning of the concentric action, which could be seen in the mirror (Figure 3).

A

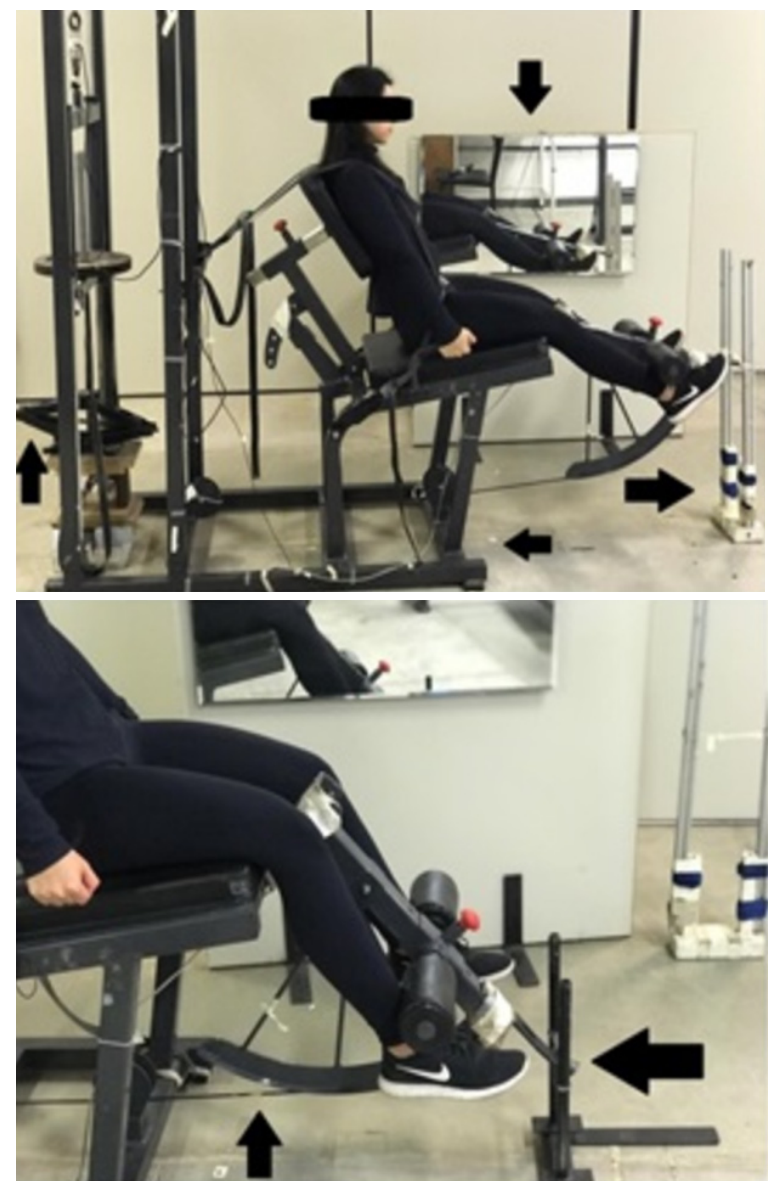

Figure 3. Structures used to control the range of motion:

(A) mirror, jack, the ground tape mark, and the metal apparatus to control the end of the concentric action at $30^{\circ}$ of knee flexion

(B) metal apparatus for the end of the concentric action at $65^{\circ}$ of knee flexion, the bell, the metal protractor, and the machine cable tape mark 
In the second session, the participants performed the $1 \mathrm{RM}$ test in 1 of the 3 proposed ROMs, following the order and procedures of the familiarization tests. The intraclass correlation coefficient $\left(\mathrm{ICC}_{3,1}\right)$ was used to check the consistency of the measurement between the results from the tests and familiarization. The $\mathrm{ICC}_{3,1}$ equalled 0.94 for INITIAL $L_{\text {ROM }}, 0.93$ for FINAL ROM $_{\text {, }}$ and 0.94 for FULL $\mathrm{ROM}_{\mathrm{ROM}}$. After the 1RM test, there was a 10-minute rest. In sequence, the participant performed 2 MVICs at $30^{\circ}$ and at $100^{\circ}$ of knee flexion (order balanced) with a 30-second and 2-minute interval between the attempts and between the angles, respectively. After the 4 MVICs (2 in each angle), another 10-minute break was given, and the volunteers performed a training protocol. The training protocol was composed of 3 sets of 7 repetitions at $60 \%$ of the weight lifted in the $1 \mathrm{RM}$ test, in the ROM specifically used on that day. Additionally, a 3-minute rest was provided between the sets. The repetition duration was determined in 4 seconds ( 2 seconds for both concentric and eccentric muscle action) with the aid of a metronome, $60 \mathrm{~b} \cdot \mathrm{min}^{-1}$. Before starting the training protocol, each volunteer performed 10 repetitions with no load, respecting the ROM and repetition duration determined. This procedure aimed at ROM and repetition duration familiarization. The training load was defined in a previous pilot study with a similar sample and ROMs. Two minutes after the training protocol execution, the volunteers performed another 2 MVICs at $30^{\circ}$ and at $100^{\circ}$ of knee flexion, following the previous procedures to verify the force reduction.

Two and 4 days after the second session, the women repeated the same procedures, but in the remaining 2 ROMs (third and fourth session, respectively). All tests were individually performed on the same day time.

Regarding the MVIC values, a load cell (type S, Tedea) with a voltage range of $+10 \mathrm{~V}$ to $-10 \mathrm{~V}$ and capacity to support up to $500 \mathrm{~kg}$ was connected in series with the machine weight support cable, with the aim to record the instantaneous force during the MVIC. For the acquisition and treatment of all signals, a specific program was used (DASYLab 11.0, Ireland) with a sampling frequency of $2000 \mathrm{~Hz}$. The load cell data were filtered with a $10-\mathrm{Hz}$ low-pass filter, second order of the Butterworth type, which allowed to obtain force information. To calculate the MVIC torque, the force value obtained by the load cell was multiplied by the distance between the point of applying force on the machine (cushion) and the machine rotation axis (aligned with the volunteer's knee). The greater MVIC torque between the 2 attempts in each condition (pre- and post-training) at each angle $\left(30^{\circ}\right.$ and $\left.100^{\circ}\right)$ was used for comparison. The $\mathrm{ICC}_{3,1}$ between the 2 MVIC torque attempts at $30^{\circ}$ and $100^{\circ}$ was 0.97 and 0.99 , respectively.

\section{Statistical procedures}

The 1RM test performance in the 3 ROMs was compared via a one-way analysis of variance (ANOVA) with repeated measures (factor 1: ROM). To compare the force reduction, absolute and relative MVIC torque values were used. For the absolute comparison, a threeway ANOVA with repeated measures was applied [factor 1: ROM (INITIAL ROM $_{\text {, FINAL }}$ ROM , and FULL ROM $_{\text {); }}$ factor 2: time (pre- and post-training protocols performance); factor 3: angle $\left(30^{\circ}\right.$ and $\left.\left.100^{\circ}\right)\right]$. The relative comparison (\%) was based on a two-way ANOVA with repeated measures [factor 1: ROM (INITIAL ROM $_{\text {, }}$ FINAL $_{\text {ROM }}$, and FULL $\left.{ }_{\text {ROM }}\right)$; factor 2: angle $\left(30^{\circ}\right.$ and $\left.100^{\circ}\right)$ ].

For all statistic tests, the significance level was set at 0.05 and, when necessary, the post-hoc Bonferroni test was used. The effect size of ANOVA was reported by eta-squared $\left(\eta^{2} ; 0.01=\right.$ small, $0.06=$ medium, 0.13 $=$ large) [20]. Complementarily, the observed power for each factor in ANOVA was also reported. In addition, the effect size of each training protocol for torque values (pre to post) was reported by Cohen's $d$ (post pre / standard deviation of pre values; trivial: $<0.20$, small: 0.20-0.60, moderate: 0.61-1.20, large: 1.21-2.0, very large: > 2.0) [21]. For all statistical analyses, the Statistical Package for the Social Sciences (SPSS 22.0) software was used. It is worth noting that previously, the repeated measure analyses: the normality, homogeneity, and sphericity of data were confirmed, respectively, by the Shapiro-Wilk, Levene, and Mauchly tests $(p>0.05)$.

\section{Ethical approval}

The research related to human use has complied with all the relevant national regulations and institutional policies, has followed the tenets of the Declaration of Helsinki, and has been approved by the ethics committee of the Federal University of Minas Gerais in Brazil (No.: 1758518.1.0000.5149).

\section{Informed consent}

Informed consent has been obtained from all individuals included in this study. 


\section{Results}

Regarding the $1 \mathrm{RM}$ test performance in the $3 \mathrm{ROMs}$, the one-way ANOVA test showed a main effect for ROM $\left(F_{2,16}=6.959 ; p=0.007\right.$; power $\left.=0.87 ; \mathrm{n}^{2}=0.21\right)$. According to the post-hoc test, the weight lifted during the 1RM test for FINAL ROM $_{\text {was }}$ similar to that for INITIAL $_{\text {ROM }}$, and both were significantly greater than the value for $\mathrm{FULL}_{\mathrm{ROM}}$. Figure 4 presents the $1 \mathrm{RM}$ test performance with each ROM.

Considering the absolute MVIC torque values, a main effect was only observed for time $\left(F_{1,8}=19.979\right.$; $p=0.002$; power $\left.=0.97 ; \eta^{2}=0.02\right)$ and angle $\left(F_{1,8}=\right.$ $43.713 ; p<0.001$; power $>0.99 ; \eta^{2}=0.38$ ). According to the post-hoc test, the pre-training torque values were

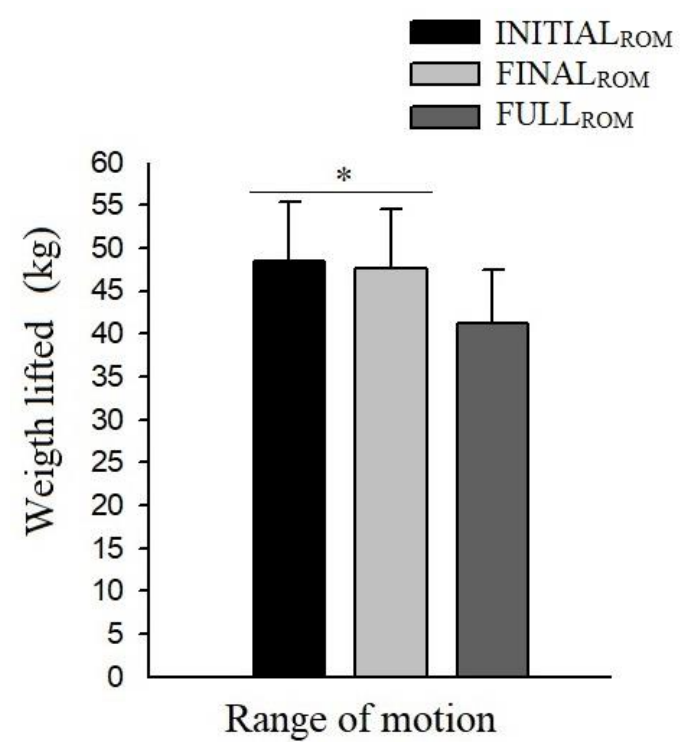

INITIAL $L_{\text {ROM }}$ - initial partial range of motion, FINAL $L_{\text {ROM }}$ - final partial range of motion, FULL ${ }_{\mathrm{ROM}}$ - full range of motion

* different from FULL ROM

Figure 4. One-repetition maximum test in 3 ranges of motion greater than the post-training torque values, and the torque value produced at $100^{\circ}$ of knee flexion was greater than that at $30^{\circ}$. No interaction or other main effect was detected by the three-way ANOVA with repeated measures. Table 1 shows the MVIC torque values before and after each training protocol in the 2 angles investigated, and the effect size by Cohen's $d$.

As for the relative MVIC torque reduction, the twoway ANOVA with repeated measures found only a main effect for angle $\left(F_{1,8}=7.053 ; p=0.029\right.$; power $=0.64$; $\left.\eta^{2}=0.02\right)$. The post-hoc test indicated that the percentage of torque reduction was greater at $30^{\circ}$ than at $100^{\circ}$ of knee flexion. No interaction effect was found. Figure 5 depicts the percentage of MVIC torque reduction for each training protocol and angles.

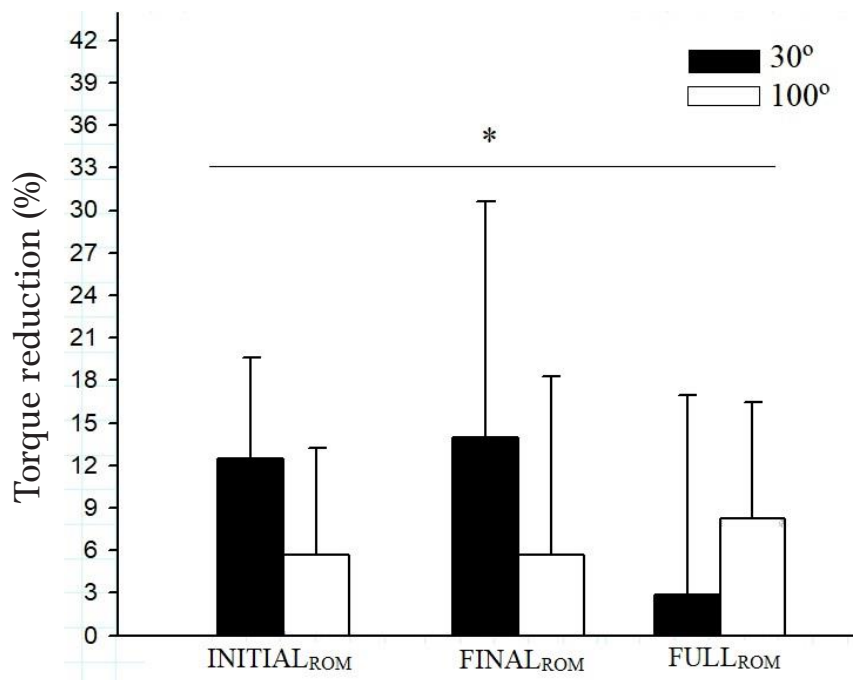

INITIAL $L_{\text {ROM }}$ - initial partial range of motion, FINAL $L_{R O M}$ - final partial range of motion, FULL ${ }_{\text {ROM }}$ - full range of motion

${ }^{*}$ greater torque reduction at $30^{\circ}$ than at $100^{\circ}$ of knee flexion (main effect)

Figure 5. Percentage of torque reduction at $30^{\circ}$ and $100^{\circ}$ of knee flexion after the performance of protocols with different ranges of motion

Table 1. Pre- and post-training isometric torque $(\mathrm{N} \cdot \mathrm{m})$ values in different ranges of motion protocols

\begin{tabular}{lcccc}
\hline \multirow{2}{*}{ Angle } & \multicolumn{3}{c}{ Training protocols } \\
\cline { 2 - 5 } & & INITIAL $_{\mathrm{ROM}}$ & FINAL $_{\mathrm{ROM}}$ & FULL $_{\text {ROM }}$ \\
\hline \multirow{2}{*}{$30^{\text {o\# }}$} & Pre* & $107.73 \pm 24.43$ & $133.32 \pm 33.06$ & $115.79 \pm 25.40$ \\
\hline Effect size (Cohen's $d$ ) & Post & $103.76 \pm 23.56$ & $110.65 \pm 17.83$ & $101.94 \pm 27.47$ \\
\hline \multirow{2}{*}{$100^{\circ}$} & & 0.16 (trivial) & 0.69 (moderate) & 0.55 (small) \\
\hline Effect size (Cohen's $d$ ) & Pre* & $168.40 \pm 33.53$ & $169.73 \pm 45.01$ & $162.47 \pm 36.56$ \\
\hline
\end{tabular}

INITIAL $_{\mathrm{ROM}}$ - initial partial range of motion, FINAL $L_{\mathrm{ROM}}$ - final partial range of motion, FULL $\mathrm{R}_{\mathrm{ROM}}$ - full range of motion

* different from post-training values, ${ }^{*}$ different from $100^{\circ}$ of knee flexion 


\section{Discussion}

The objective of the present study was to compare the performance in the $1 \mathrm{RM}$ test in 3 different ROMs in women, and the force reduction at $30^{\circ}$ and $100^{\circ}$ after the performance of training protocols with different ROMs (the same as in the 1RM tests). This is the first study that compared the 1RM test results obtained in the INITIAL ROM $_{\text {, FINAL }}$ ROM, and FULL ROM $_{\text {, }}$, and the force reduction in different knee joint angles after a training session in the NITIAL ROM $_{\text {, FINAL }}$ ROM, and FULL ROM $_{\text {. }}$. According to the main results, there was no difference in the $1 \mathrm{RM}$ test performance between the INITIAL $\mathrm{ROM}_{\mathrm{RO}}$ and FINAL ${ }_{\text {ROM }}$, and these 2 ROMs presented greater

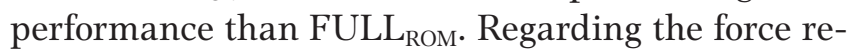
duction, the performance of training the protocols differentiated by ROM provoked significant reductions at $30^{\circ}$ and $100^{\circ}$, with no difference among the ROMs. In addition, the force reduction at $30^{\circ}$ was greater than at $100^{\circ}$.

The INITIAL ROM $_{\text {and }}$ FINAL ROM $_{\text {pesented similar }}$ $1 R M$ test values, which could be explained by biomechanical factors. It has been seen that at ca. $70^{\circ}$ of knee flexion $\left(0^{\circ}=\right.$ knee fully extended $)$, the muscle is found at or near optimal length for torque production in a knee extension machine [22]. In this case, when the volunteers started the 1 RM test in the FINAL ROM $\left(65^{\circ}\right.$ of knee flexion), the quadriceps femoris muscle was near the optimal length to produce force in comparison with the condition of INITIAL $\mathrm{ROM}_{\mathrm{ROM}}\left(100^{\circ}\right.$ of knee flexion). Thus, a greater initial acceleration might have been imposed to leave the inertia for FINAL $\mathrm{ROM}_{\mathrm{RO}}$ than for INITIAL $_{\text {ROM }}$, which facilitated overcoming the overload in the following angles.

However, although at FINAL $_{\text {ROM }}$, the test started with a better muscle length to produce force than at INITIAL $_{\text {ROM }}$, the volunteers encountered a greater external arm moment than at INITIAL ${ }_{\mathrm{ROM}}$ [3]. An increase of the external arm moment demands an increase in the force production [23]. Thus, despite the volunteers started the concentric action at a better muscle length to produce force with FINAL $L_{\text {ROM }}$ than with INITIAL $L_{\text {ROM }}$, the external arm moment was also greater with FINAL $_{\text {ROM }}$. On the other hand, with INITIAL ROM $_{\text {, the }}$ volunteers started the $1 \mathrm{RM}$ test with a poorer force production capacity, but encountering a shorter external arm moment than with FINAL ROM $_{\text {. The combina- }}$ tion of greater or lower torque capacity at the beginning of the concentric action with a greater or shorter external arm moment at the end of the concentric action for FINAL ${ }_{\text {ROM }}$ and INITIAL ${ }_{\text {ROM }}$, respectively, balanced the 1RM test results between these ROMs.
Conversely, with FULL $\mathrm{ROM}_{\text {, }}$, the women lifted significantly less weight than with the other 2 ROMs. The sum of the angles from INITIAL ${ }_{\text {ROM }}$ and FINAL ROM being performed sequentially indicates that the participants had to initiate the concentric action with lesser torque production capacity than with FINAL $\mathrm{ROM}_{\mathrm{RO}}$, and had to overcome a greater external arm moment at the final angles of ROM than with INITIAL $\mathrm{R}_{\mathrm{ROM}}$. Moreover, the rise in angular distance excursed led to an increase of the mechanical work (force $\times$ displacement). It has been observed that an increase in the mechanical work diminishes the ability to sustain a force task [24], which helps explain why the women lifted less weight at FULL ROM $_{\text {than }}$ at the other 2 ROMs.

Regarding the force reduction results, the main effect for time showed that the training protocols performed in the 3 ROMs were similarly efficient to reduce the force production in the angles tested. Under fatigue conditions, the force reduction could be associated with many physiological alterations [25]. The accumulation of intracellular hydrogen ions may impair the function of the contractile proteins: ionic changes in the action potential and failure of sarcoplasmic reticulum $\mathrm{Ca}^{2+}$ release are factors associated with fatigue [13]. In addition, a greater force depression was noticed at $30^{\circ}$ of knee flexion in comparison with $100^{\circ}$. It means that the force production capacity at $30^{\circ}$ was more affected by the training protocols than at $100^{\circ}$.

Although the present study did not aim to provide information regarding possible mechanisms involved in force reduction response, it is possible to speculate that the physiological differences of contracting in different muscle lengths may have caused the distinct force reduction responses between angles. When a muscle is lengthened, the $\mathrm{Ca}^{2+}$ sensitivity increases [26]. Thus, the force reduction due to the decrease of $\mathrm{Ca}^{2+}$ release in fatigue conditions would be minimized when the muscle is demanded to contract at a bigger length than at a smaller one. This suggestion is supported by the findings by MacNaughton et al. [27], who demonstrated a greater decrease of active force at a small muscle length in comparison with optimal muscle length. Moreover, at a bigger muscle length, less overlapping of actin and myosin is expected to occur than at a smaller muscle length [10]. Less overlapping of actin and myosin means that less adenosine triphosphate becomes hydrolysed and, consequently, less accumulation of metabolic ions related to muscle fatigue $\left(\mathrm{P}_{\mathrm{i}}, \mathrm{P}_{\mathrm{cr}}, \mathrm{H}^{+}\right)$ occurs [17]. This reasoning could help explain why greater force reduction was observed at $30^{\circ}$ than at $100^{\circ}$ of knee flexion; however, it is speculative. 
Another explanation is perhaps linked to the findings by Joumaa et al. [28], who demonstrated a change in the actin filament conformational structure after active shortening, which could inhibit the cross-bridge attachment and muscle stiffness. According to Chen et al. [25], the reduction of the number of cross-bridges after active contractions is the main factor in force reduction. Thus, the decrease of the number of crossbridge attachments after the training protocols, caused by the change in the actin structure, may have influenced muscle force production more at $30^{\circ}$ of knee flexion (smaller muscle length) than at $100^{\circ}$ (bigger muscle length) as the passive force offers greater force contribution at a bigger muscle length than at a smaller one [10]. In this case, perhaps a greater time for recovering of the maximum force production at a smaller than at a bigger muscle length would be necessary after resistance training, regardless of the ROM adopted, but further studies are required to clarify this issue.

Nevertheless, the production of the knee extension torque is derived from the sum of the torque produced from each quadriceps muscle [3], and the change in the knee joint angle may impact on the length and the torque production of each muscle differently [12]. Akima et al. [11] demonstrated greater muscle fatigue (decrease of electromyographic median frequency) of the vastus lateralis muscle during 40 isometric contractions with the knee flexed $\left(100^{\circ}\right)$ than extended $\left(40^{\circ}\right)\left(0^{\circ}=\right.$ knee full extended). This response was not followed by the other muscles; e.g., the rectus femoris muscle presented similar fatigue during contractions in either angle. Further investigation is needed to verify whether individual muscle torque production is influenced by dynamic contractions in different ROMs.

No interaction between the factors of angle and ROM was found. It was expected that greater force depression would occur at the angles excursed during the training with the protocols. This expectation was based on the results of studies that showed a specific force increase only at or near the angles trained [5, 15, 16]. However, it has been reported that fatigue is not a necessary stimulus for strength gains [29, 30], and perhaps the angle-specific force increase is not linked to fatigue [30]. Our results support this rationale as anglespecific fatigue was not seen in the present study, but a longitudinal study is needed to confirm this line of reasoning.

Other results from ANOVA revealed that greater isometric force was produced at $100^{\circ}$ than at $30^{\circ}$ of knee flexion. This is in line with another study [11]. At $100^{\circ}$ of knee flexion, a greater passive force can be produced and transmitted by the muscle-tendon unit than at $30^{\circ}$, contributing to the achievement of a greater amount of force production from the sum of passive and active forces $[8,11]$.

Notwithstanding, the results from the present study refer to females, and may not be extrapolated to men owing to the physiological and biomechanical differences between genders [14], specially under fatigue [8]. This is a limitation of the study, and further research needs to be conducted in men to improve the understanding of the effect of ROM on strength performance.

\section{Conclusions}

The present study showed that the performance of training protocols differentiated by ROM led to similar isometric force reduction, regardless of the angle tested. Besides, greater force reduction occurred at $30^{\circ}$ than at $100^{\circ}$ of knee flexion, irrespective of the training protocol, and greater amount of weight was lifted in the 1RM test in the training protocols with partial ROMs as compared with FULL ROM $_{\text {. }}$

\section{Disclosure statement}

No author has any financial interest or received any financial benefit from this research.

\section{Conflict of interest}

The authors state no conflict of interest.

\section{References}

1. Newmire DE, Willoughby DS. Partial compared with full range of motion resistance training for muscle hypertrophy: a brief review and an identification of potential mechanisms. J Strength Cond Res. 2018;32(9): 2652-2664; doi: 10.1519/JSC.0000000000002723.

2. Guex K, Degache F, Morisod C, Sailly M, Millet GP. Hamstring architectural and functional adaptations following long vs. short muscle length eccentric training. Front Physiol. 2016;7:340; doi: 10.3389/fphys.2016. 00340.

3. Anderson DE, Madigan ML, Nussbaum MA. Maximum voluntary joint torque as a function of joint angle and angular velocity: model development and application to the lower limb. J Biomech. 2007;40(14):31053113; doi: 10.1016/j.jbiomech.2007.03.022.

4. Dalleau G, Baron B, Bonazzi B, Leroyer P, Verstraete T, Verkindt $\mathrm{C}$. The influence of variable resistance moment arm on knee extensor performance. J Sports Sci. 2010; 28(6):657-665; doi: 10.1080/02640411003631976.

5. Bloomquist K, Langberg H, Karlsen S, Madsgaard S, Boesen M, Raastad T. Effect of range of motion in heavy load squatting on muscle and tendon adaptations. Eur J Appl Physiol. 2013;113(8):2133-2142; doi: 10.1007/ s00421-013-2642-7. 
6. Rhea MR, Kenn JG, Peterson MD, Massey D, Simão R, Marin PJ, et al. Joint-angle specific strength adaptations influence improvements in power in highly trained athletes. Hum Mov. 2016;17(1):43-49; doi: 10.1515/ humo-2016-0006.

7. Martínez-Cava A, Hernández-Belmonte A, CourelIbáñez J, Morán-Navarro R, González-Badillo JJ, Pallarés JG. Bench press at full range of motion produces greater neuromuscular adaptations than partial executions after prolonged resistance training. J Strength CondRes.2019; doi: 10.1519/JSC.0000000000003391.

8. El-Ashker S, Allardyce JM, Carson BP. Sex-related differences in joint-angle-specific hamstring-to-quadriceps function following fatigue. Eur J Sport Sci. 2019; 19(8):1053-1061; doi: 10.1080/17461391.2019.1574904.

9. Kooistra RD, Blaauboer ME, Born JR, de Ruiter CJ, de Haan A. Knee extensor muscle oxygen consumption in relation to muscle activation. Eur J Appl Physiol. 2006; 98(6):535-545; doi: 10.1007/s00421-006-0298-2.

10. MacIntosh BR, MacNaughton MB. The length dependence of muscle active force: considerations for parallel elastic properties. J Appl Physiol. 2005;98(5):1666-1673; doi: 10.1152/japplphysiol.01045.2004.

11. Akima H, Tomita A, Ando R. Effect of knee joint angle on the neuromuscular activation of the quadriceps femoris during repetitive fatiguing contractions. J Electromyogr Kinesiol. 2019;49:102356; doi: 10.1016/j. jelekin.2019.102356.

12. Kooistra RD, de Ruiter CJ, de Haan A. Muscle activation and blood flow do not explain the muscle lengthdependent variation in quadriceps isometric endurance. J Appl Physiol. 2005;98(3):810-816; doi: 10.1152/japplphysiol.00712.2004.

13. Allen DG, Lamb GD, Westerblad H. Skeletal muscle fatigue: cellular mechanisms. Physiol Rev. 2008;88(1): 287-332; doi: 10.1152/physrev.00015.2007.

14. Raeder C, Wiewelhove T, De Paula Simola RÁ, Kellmann M, Meyer T, Pfeiffer M, et al. Assessment of fatigue and recovery in male and female athletes after six days of intensified strength training. J Strength Cond Res. 2016;30(12):3412-3427; doi: 10.1519/JSC.00000 00000001427.

15. Graves JE, Pollock ML, Jones AE, Colvin AB, Leggett $\mathrm{SH}$. Specificity of limited range of motion variable resistance training. Med Sci Sports Exerc. 1989;21(1):84-89; doi: 10.1249/00005768-198902000-00015.

16. Noorkõiv M, Nosaka K, Blazevich AJ. Neuromuscular adaptations associated with knee joint angle-specific force change. Med Sci Sports Exerc. 2014;46(8):15251537; doi: 10.1249/MSS.0000000000000269.

17. Rooney KJ, Herbert RD, Balnave RJ. Fatigue contributes to the strength training stimulus. Med Sci Sports Exerc. 1994;26(9):1160-1164.

18. Beck TW. The importance of a priori sample size estimation in strength and conditioning research. J Strength Cond Res. 2013;27(8):2323-2337; doi: 10.1519/JSC. 0b013e318278eea0.
19. Fukutani A, Joumaa V, Herzog W. Influence of residual force enhancement and elongation of attached crossbridges on stretch-shortening cycle in skinned muscle fibers. Physiol Rep. 2017;5(22):e13477; doi: 10.14814/ phy2.13477.

20. Cohen J. Statistical power analysis for the behavioral sciences. New York: Lawrence Erlbaum Associates; 1988.

21. Batterham AM, Hopkins WG. Making meaningful inferences about magnitudes. Int J Sports Physiol Perform. 2006;1(1):50-57; doi: 10.1123/ijspp.1.1.50.

22. Blazevich AJ, Cannavan D, Coleman DR, Horne S. Influence of concentric and eccentric resistance training on architectural adaptation in human quadriceps muscles. J Appl Physiol. 2007;103(5):1565-1575; doi: 10.1152/japplphysiol.00578.2007.

23. Bakenecker P, Raiteri B, Hahn D. Patella tendon moment arm function considerations for human vastus lateralis force estimates. J Biomech. 2019;86:225231; doi: 10.1016/j.jbiomech.2019.01.042.

24. Kosterina N, Westerblad H, Lännergren J, Eriksson A. Muscular force production after concentric contraction. J Biomech. 2008;41(11):2422-2429; doi: 10.1016/j. jbiomech.2008.05.019.

25. Chen J, Hahn D, Power GA. Shortening-induced residual force depression in humans. J Appl Physiol. 2019; 126(4):1066-1073; doi: 10.1152/japplphysiol.00931.2018.

26. MacIntosh BR. Recent developments in understanding the length dependence of contractile response of skeletal muscle. Eur J Appl Physiol. 2017;117(6):1059-1071; doi: 10.1007/s00421-017-3591-3.

27. MacNaughton MB, Campbell JJ, MacIntosh BR. Dantrolene, like fatigue, has a length-dependent effect on submaximal force-length relationships of rat gastrocnemius muscle. Acta Physiol. 2007;189(3):271-278; doi: 10.1111/j.1748-1716.2006.01645.x.

28. Joumaa V, Curtis Smith I, Fakutani A, Leonard T, Ma W, Irving $\mathrm{T}$, et al. Evidence for actin filament structural changes after active shortening in skinned muscle bundles. Biophys J. 2018;114(3 Suppl. 1):135a; doi: 10.1016/j.bpj.2017.11.765.

29. Folland JP, Irish CS, Roberts JC, Tarr JE, Jones DA. Fatigue is not a necessary stimulus for strength gains during resistance training. Br J Sports Med. 2002;36(5): 370-373; doi: 10.1136/bjsm.36.5.370.

30. Pedersen KK, Madsen MK, Hvid LG, Overgaard K. Concentric strength training at optimal or short muscle length improves strength equally but does not reduce fatigability of hamstring muscles. Physiol Rep. 2019; 7(16):e14196; doi: 10.14814/phy2.14196. 